\title{
EDITORIAL
}

\section{Coarctation of the aorta in adults: do we need surgeons?}

\section{J Mullen}

\begin{abstract}
With the introduction of endovascular stents, catheter based intervention in the treatment of aortic coarctation now represents a viable alternative to surgery
\end{abstract}

of recoil is inevitable following balloon dilatation and the obstruction may not be adequately relieved, resulting in a high rate of reintervention, particularly in non-discrete coarctation. In early studies this may have led to overdilatation, explaining the high incidence of aneurysm formation reported to occur in between $5-20 \%$ of cases following balloon angioplasty ${ }^{8}$ and occasional catastrophic and fatal disruption of the aorta. Despite this, results comparable with that achieved at surgery have been reported. ${ }^{8}{ }^{10}$ More recently, with improvements in technology, endovascular stents have been used to treat coarctation. ${ }^{11-18}$ Unlike balloon dilatation, stents utilise the elastic properties of the aortic wall to affect an increase in luminal diameter, which would otherwise recoil. While in children their use is limited by the size of current delivery systems and the intrinsic need for redilatation in line with somatic growth, in older children and adults stents represent a viable alternative to surgery.

\section{TRANSCATHETER TREATMENT}

In this issue of Heart Zabal and colleagues report their experience of transcatheter treatment of native coarctation in adults, ${ }^{19}$ adding to the considerable literature on balloon dilatation ${ }^{8}$ and the growing data on endovascular stents. ${ }^{11-18}$ Their data provide further evidence to support transcatheter treatment of native coarctation as a safe and effective alternative to surgery in adults.

In accord with previous studies, ${ }^{10}$ Zabal and colleagues found that patients with hypoplasia of the arch or isthmus or with a tubular coarctation responded poorly to angioplasty alone. By contrast for discrete coarctation, they report excellent results using balloon angioplasty and propose an algorithm for treatment whereby stents are reserved for cases where this fails to reduce the gradient $<10 \mathrm{~mm} \mathrm{Hg}$. Although attractive, it is important to appreciate that this paradigm has not been tested and the case for initial balloon angioplasty versus primary stenting in all cases remains unproven. Zabal and colleagues, ${ }^{19}$ like others, defined a significant coarctation and success of the intervention as a gradient $<20 \mathrm{~mm} \mathrm{Hg}$. However, cardiovascular risk may be increased even in those with mild coarctation, not deemed sufficient to warrant intervention, and there are no data to indicate that reduction in the gradient below any particular threshold will confer clinical benefit. In this context, it is important to note that stenting resulted in a greater reduction in gradient than following angioplasty, and a lower incidence of hypertension at follow up. There are other reasons to consider primary stenting in adults,
Royal Brompton Hospita,

Sydney Street, London

SW3 6NP, UK

m.mullen@

rbh.nthames.nhs.uk disruption of the aortic intima and media. ${ }^{9}$ As a result its use in native coarctation, where the artery is already intrinsically weakened by cystic medial necrosis, remains controversial. A degree 
where somatic growth is not an issue. For many operators, concern remains that further instrumentation of the aorta after failed balloon dilatation might provoke more extensive dissection and increase the risk of a subsequent stent malposition. Stents may reduce trauma to the vessel wall through dispersion of the forces over a larger area, control small dissections, and reduce the likelihood of aneurysm formation. The two cases of aneurysm formation that were noted in the series by Zabal and colleagues ${ }^{19}$ both occurred after balloon dilatation, one in a patient with a discrete coarctation.

\section{SO ARE STENTS THE ANSWER?}

Of the 320 cases reported in series to date, ${ }^{11-19} 175$ have been in patients with native coarctation including both children and adults. Generally excellent results have been achieved with clinical effectiveness as judged by almost complete abolishment of the gradient in $>95 \%$. Restenosis does not appear to be a major problem during follow up and in a number of patients further dilatation has been effective for residual stenosis. Stents also appear to effectively treat severe complex coarctations with hypoplasia of the isthmus and transverse arch and long tubular stenosis. Although intuitively these patients seem less ideal for a transcatheter treatment, in adults, surgical repair is also challenging and will often necessitate the use of extra-anatomic aorto-aorto bypass (an option that would still be feasible should a transcatheter approach fail).

Despite the obvious trauma induced to the aortic wall, major complications appear to be rare. To date there have been no deaths reported, with a need for emergency surgery in less than $1 \%$ of cases. The inherent bias in reporting such series might underestimate the risk of serious complications and I am aware of at least two deaths during coarctation stenting. Late aneurysm formation has been reported in $4 \%$ of cases, though their true incidence is unknown and will undoubtedly depend not only on the technique used but the rigour with which their presence is sought. The sensitivity of plain radiology and echocardiography alone for detecting aneurysm formation is less than $100 \%$ and that of echocardiography likely to be reduced even further by the presence of a metallic stent. It is our practice to routinely perform a contrast enhanced spiral computed tomographic scan at six weeks post-stenting, with a further assessment at one year. Other complications include balloon rupture, malposition of the stent in $4 \%$, vascular complications at the femoral artery access site in $3 \%$, and myocardial infarction and stroke in one patient each. Many of these complications are avoidable with increased experience, both generally and for individual operators, and with the development of new stents with rounded edges and less shortening characteristics, ${ }^{17}$ smaller and better balloons and delivery systems, and arterial closure devices. Major complications, although rare, are dramatic and life threatening, though with the appropriate array of equipment may usually be treated in the catheter laboratory without recourse to surgery. For this reason the procedure should be limited to centres with on site cardiac surgery, a sufficient case load to maintain adequate institutional and operator experience, and the necessary stock of balloon catheters, stents, and delivery systems.

\section{UNCERTAINTIES IN STENTING}

Many questions remain unanswered and further collaborative prospective research is essential to refine the techniques of stenting and define its limitations and long term benefit. A number of operators have promoted the use of covered stents for native coarctation, to reduce the risk of dissection and aneurysm formation. ${ }^{20}$ Staged dilatation, allowing healing to occur between procedures or in line with somatic growth, is intuitively attractive and appears feasible in humans. However, in one study in an animal model this was associated with a high incidence of aortic rupture and caution needs to be observed. In many centres it is usual practice to use larger balloons to "flare" stents within dilated pre- or post-stenotic segments, improving apposition of the stent with the aortic wall and promoting endothelialisation. Paradoxically, this practice might increase the risk of dissection, forcing the ends of the stent into the aortic media. Further data are needed on the effect of the introduction of a rigid stent on vascular haemodynamics. Undoubtedly blood pressure control is improved and Marshall and colleagues ${ }^{13}$ have reported a beneficial effect on left ventricle end diastolic pressure, but the short follow up and few patients precludes data on hard clinical outcomes. Pihkala and colleagues ${ }^{21}$ reported no adverse effect of the implantation of a stent in the normal aorta of a pig, but this cannot imply that stenting will reverse the reduced compliance seen in upper body elastic arteries in human coarctation ${ }^{22}$ or that the presence of a rigid stent might in itself alter vascular haemodynamics in an adverse way, particularly during exercise. Caution should also be observed with regard to the generally short follow up and the effect of the stent on the aortic wall. Loss of exposure to the normal pulsatile movement alters the phenotype of vascular smooth muscle and connective tissue, increasing apoptosis, which might promote aneurysm formation. ${ }^{23}$

\section{SUMMARY}

The short term results of the transcatheter treatment of aortic coarctation appear excellent. This is particularly the case in adults where operative risk is increased and the use of endovascular stents appears to be safe and highly effective. However, case numbers are small and it is important to remain vigilant, with long term event-free palliation and reduction in cardiovascular risk the only truly valid end points. Many patients will hope to live with their stent for 50-60 years or more and should be counselled appropriately about the potential for late complications. Surgery remains a viable alternative for children and those who do not want to take this risk as, should it be necessary, surgical repair following stenting is likely to be more difficult and carry a higher risk, and neither our patients nor the surgeons will thank us.

The introduction of any new technique demands careful evaluation of its efficacy, risk, convenience or cost with reference to the standard treatment. While the jury is still out for catheter based intervention in aortic coarctation, so far the evidence looks good.

\section{REFERENCES}

1 Maron BJ, Humphries JO, Rowe RD, et al. Prognosis of surgically corrected coarctation of the aorta. A 20-year postoperative appraisal. Circulation 1973;47:1 19-26.

2 Campbell M. Natural history of coarctation of the aorta. Br Heart J 1970;32:633-40.

3 Liberthson RR, Pennington DG, Jacobs ML, et al. Coarctation of the aorta: review of 234 patients and clarification of management problems. Am J Cardiol 1979;43:835-40.

4 Cohen M, Fuster V, Steele PM, et al. Coarctation of the aorta. Long-term follow-up and prediction of outcome after surgical correction. Circulation 1989:80:840-5.

5 Aris A, Subirana MT, Ferres $P$, et al. Repair of aortic coarctation in patients more than 50 years of age. Ann Thorac Surg 1999;67:1376-9.

6 Bouchart F, Dubar A, Tabley A, et al. Coarctation of the aorta in adults: surgical results and long-term follow-up. Ann Thorac Surg 2000;70:1483-8.

7 Connolly JE. Hume memorial lecture. Prevention of spinal cord complications in aortic surgery. Am J Surg 1998;176:92-101.

8 Ovaert C, Benson LN, Nykanen D, et al. Transcatheter treatment of coarctation of the aorta: a review. Pediatr Cardiol 1998;19:27-44

9 Sohn S, Rothman A, Shiota T, et al. Acute and follow-up intravascular ultrasound findings after balloon dilation of coarctation of the aorta. Circulation 1994;90:340-7. 
10 Ovaert C, McCrindle BW, Nykanen D, et al. Balloon angioplasty of native coarctation: clinical outcomes and predictors of success. J Am Coll Cardiol 2000;35:988-96

11 Bulbul ZR, Bruckheimer E, Love JC, et al. Implantation of balloon-expandable stents for coarctation of the aorta: implantation data and short-term results. Cathet Cardiovasc Diagn 1996;39:36-42.

12 Thanopoulos BD, Hadjinikolaou L, Konstadopoulou GN, et al. Stent treatment for coarctation of the aorta: intermediate term follow up and technical considerations. Heart 2000;84:65-70.

13 Marshall AC, Perry SB, Keane JF, et al. Early results and medium-term follow-up of stent implantation for mild residual or recurrent aortic coarctation. Am Heart J 2000;139: 1054-60.

14 Suarez dL, Pan M, Romero M, et al. Immediate and follow-up findings after stent treatment for severe coarctation of aorta. Am J Cardiol 1999:83:400-6.

15 Harrison DA, Mclaughlin PR, Lazzam C, et al. Endovascular stents in the management of coarctation of the aorta in the adolescent and adult: one year follow up. Heart 2001;85:561-6.

16 Duke C, Qureshi SA. Aortic coarctation and recoarctation: to stent or not to stent? J Interv Cardiol 2001;14:283-98.
17 Cheatham JP. Stenting of coarctation of the aorta. Catheter Cardiovasc Interv 2001;54:112-25.

18 Ebeid MR, Prieto LR, Latson LA. Use of balloon-expandable stents for coarctation of the aorta: initial results and intermediate-term follow-up. I Am Coll Cardiol 1997;30:1847-52.

19 Zabal C, Attie F, Buendía-Hernández, et al. The adult patient with native coarctation of the aorta: ballon angioplasty or primary stenting? Heart 2003:89:77-83.

20 de Giovanni JV. Covered stents in the treatment of aortic coarctation. J Interv Cardiol 2001;14;187-90.

21 Pihkala J, Thyagarajan GK, Taylor GP, et al. The effect of implantation of aortic stents on compliance and blood flow. An experimental study in pigs. Cardiol Young 2001;11:173-81.

22 de Divitiis $M$, Pilla $C$, Kattenhorn $M$, et al. Vascular dysfunction after repair of coarctation of the aorta: impact of early surgery. Circulation 2001;104:1165-70.

23 Kollum M, Kaiser S, Kinscherf R, et al. Apoptosis after stent implantation compared with balloon angioplasty in rabbits. Role of macrophages. Arterioscler Thromb Vasc Biol 1997; 17:2383-8.

\section{IMAGES IN CARDIOLOGY}

\section{Aortic dissection extending to both carotid arteries}

A 49 year old man collapsed and developed convulsions after complaining about breathlessness. On initial examination the patient was hypotensive, stuporous, with no reaction to painful stimulus on the left side. A cerebral computerised tomography suggested a right occipital ischaemic lesion, and the patient was transferred to our institution. Upon arrival to our hospital, the patient was intubated, non-sedated, and had no reaction to painful stimuli on the right side, as well as a lack of brain stem reflexes. He was hypotensive despite treatment with catecholamines. The ECG showed pronounced anterolateral ST segment depression and biphasic T waves, consistent with severe myocardial ischaemia; chest $x$ ray findings were consistent with acute lung oedema. Transoesophageal echocardiography showed a type A aortic dissection with a long intimal flap (panel A, arrowheads: Ao, aorta; LA, left atrium; LV, left ventricle; RV, right ventricle), protruding into the left ventricle during
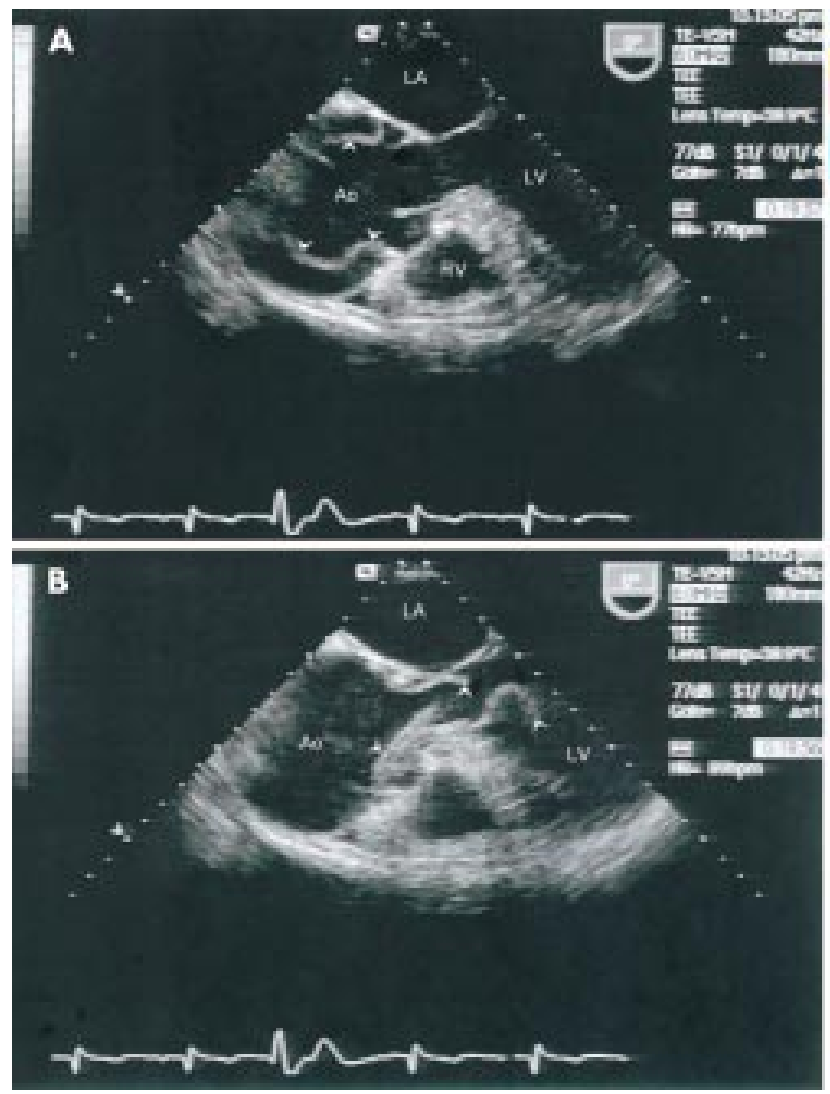

diastole (panel B, arrowheads), and as a consequence severe aortic regurgitation (panel $\mathrm{C}$ ). Duplex sonography could not demonstrate any flow in the right common carotid artery. Cardiac surgery was not carried out because of the poor neurological prognosis, and the patient died a few hours later.

Necropsy showed a type A aortic dissection with antegrade extension of the intimal flap in both carotid arteries and almost complete occlusion of the lumina (panel D). The left main coronary artery was found to be occluded by a flail retrograde intimal flap.

M Rotter

M Pavlicek

E Delacretaz etienne.delacretaz@insel.ch

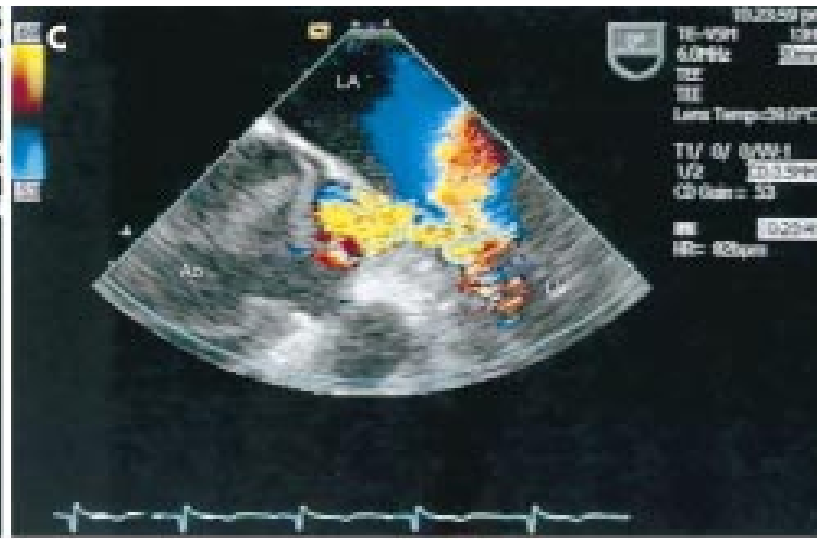

D

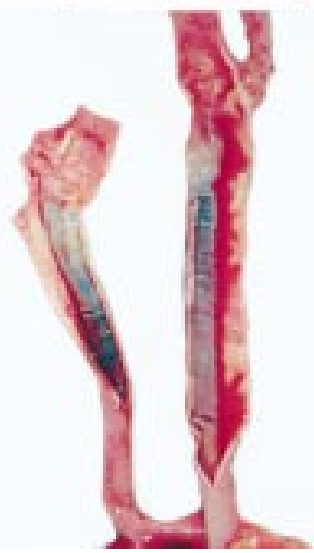

